\title{
Herbal Medicine for Prevention and Therapy in Breast Cancer
}

\author{
Mohd Fadzelly Abu Bakar $\mathbb{D},{ }^{1}$ Azis Saifudin $\mathbb{D}^{2},{ }^{2}$ Peng Cao $\mathbb{D}^{3},{ }^{3}$ and Norhaizan Mohd Esa $\mathbb{D}^{4}$ \\ ${ }^{1}$ Faculty of Applied Sciences and Technology, Universiti Tun Hussein Onn Malaysia (UTHM)-Pagoh Campus, Muar 84600, \\ Johor, Malaysia \\ ${ }^{2}$ Faculty of Pharmacy, Universitas Muhammadiyah Surakarta, Jalan A. Yani, Tromol Pos 1, Pabelan Kartasura, Surakarta, \\ Central Java, Indonesia \\ ${ }^{3}$ Department of Pharmacology, School of Pharmacy, Nanjing University of Chinese Medicine, Nanjing 210023, China \\ ${ }^{4}$ Department of Nutrition, Faculty of Medicine and Health Sciences, Universiti Putra Malaysia, Serdang 43400, \\ Selangor, Malaysia \\ Correspondence should be addressed to Mohd Fadzelly Abu Bakar; fadzelly@uthm.edu.my
}

Received 28 July 2021; Accepted 28 July 2021; Published 15 August 2021

Copyright (C) 2021 Mohd Fadzelly Abu Bakar et al. This is an open access article distributed under the Creative Commons Attribution License, which permits unrestricted use, distribution, and reproduction in any medium, provided the original work is properly cited.

Cancer is becoming more common globally. Breast cancer is one of the most prevalent forms of cancer and one of the main causes of cancer mortality. While modern medicine has been used as the primary cure for this disease, traditional herbal medicine has been commonly used as a complementary and alternative strategy in some developed and developing countries.

To provide scientific justification for the use of herbal medicine, it is necessary to record and publish preclinical and clinical evidence-based research. Concerns with herbal medicine including the consistency and standardisation of the herbal product (particularly the presence of bioactive compounds responsible for the effect), effectiveness, mode of action, safety, and herb-drug interaction if used as complementary and/or alternative medicine need to be further explored.

This special issue seeks to provide a forum for researchers to publish the most recent advances and clinical studies in the use of herbal medicines for breast cancer prevention and treatment, including ethnopharmacology, natural product chemistry, effectiveness, safety, dosage and toxicity, in vitro and in vivo preclinical trials, herb-drug relationship, clinical trials, and potential biochemical and molecular pathways. This special issue included fifteen articles which were carefully reviewed and accepted for publication and briefly described as follows.

Some of the papers of this special issue focus on plant extracts from different countries of the world, and their potential to treat and prevent breast cancer using in vitro and/or in vivo methods. In some cases, a new generation of ICT technique (i.e., network pharmacology and molecular docking) was used to determine the potential plant phytochemicals and their possible mechanism of action to treat and/or prevent the incidence of breast cancer. In addition to that, in vitro and in vivo studies were also being conducted to confirm the anticancer activity from the network pharmacology results. In some cases, a partial phytochemical isolation and further characterization was performed. One clinical trial was conducted to determine the efficacy of herbal medicines to treat and/or prevent breast cancer in human patients. Of these, several works involve herbal preparations from single and/or polyherbal formulations from standardized herbal traditional Chinese medicine.

$\mathrm{W}$. Chen et al. investigated the anticancer potential of herbal traditional Chinese medicine Fuzheng Yiliu formula (FZYLF) against MDA-MB-231/Adr cell line with high invasive ability and multidrug-resistant breast cancer cells. Using in vitro (established cancer cells) and in vivo (MDAMB-231/Adr tumor xenografted in nude mice) approaches, they conclude that FZYLF can inhibit the invasion and metastasis as well as induce cytotoxicity in MDA-MB-231/ Adr human breast cancer cells. They propose the regulation of WAVE3 expression as the possible molecular mechanism of action of FZYLF as anticancer agent.

L. B. Moses et al. investigated the anticancer capacity of Eurycoma longifolia (Jack.) or locally known as "Tongkat 
Ali" leaf extracts and their probable anticancer mode of action in vitro against non-hormone-dependent MDA-MB231 and hormone-dependent MCF-7 breast cancer cell lines. The leaves of E. longifolia were converted into both unfermented and fermented products, which were then screened with their action against both cancer cell lines. Flow cytometry was used to investigate apoptotic cell quantification, cell cycle distribution, and the expression of caspases and apoptotic proteins. MDA-MB-231 cells had substantial activities of cytochrome c, caspase-3, Bax, and Bcl-2 apoptotic proteins, while MCF-7 cells had significant activities of caspase-8, cytochrome c, Bax, p53, and Bcl-2 apoptotic protein. This extract has the highest levels of phenolics such as gallic acid, chlorogenic acid, ECG, and EGCG, which can contribute to its potent anticancer action.

The secondary metabolites, antioxidant, and antiproliferative activity of Dioscorea bulbifera leaves collected from Endau Rompin, Johor, Malaysia, was explored by M. M. Mainasara et al. The results showed that the plant extracts displayed high antioxidant activity and induced cytotoxicity in both MCF-7 and MDA-MB-231 breast cancer cell lines. Apoptosis and cell cycle arrest have been shown to be part of the mechanism of action. These authors found that the plant contains at least of 39 metabolites that might synergistically contribute to the bioactivity.

C. Zhu et al. reported the anticancer potential of berberine (BBR), a kind of isoquinoline alkaloid that is extracted from Coptidis Rhizoma or Huanglian. BBR has been shown to display diverse health benefit properties such as antimicrobial, cardioprotective, and antidiabetic properties. They investigate the suppressive abilities of BBR on both MCF-7 and MDA-MB-231 breast cancer cells and confirm its underlying mechanisms with miR-214-3p. They found that BBR has the potential to suppress the proliferation of MCF-7 and MDA-MB-231 breast cancer cells by upregulating the expression of miR-214-3p and increasing its inhibition to stem cell transplant (SCT). As a conclusion, miR-214-3p/SCT axis is a potential therapeutic target in the mechanism of BBR to suppress breast cancer.

T. Tuy-on et al. investigated the anticancer potential of selected Thai traditional cancer remedies to induce cytotoxicity in breast, cervical, and ovarian cancer cells in vitro. The hierarchical cluster analysis (HCA) was used to classify the extracts by their cytotoxic characteristics. They design this method to predict the correlation between the plant's bioactive compounds and biological activities. The results showed that eleven plants normally used in Thai traditional medicine (TTM) were active against at least one of the cancer cell lines, while about $2 / 3$ of all extracts were active against all tested cell lines. The remaining plant extracts might not be considered as active but may be needed as complementary medicine according to the TTM theory. They suggested further studies (i.e., in vivo work) to determine the exact efficacy of the herbal remedies.

Carvacrol is a phenol monoterpene and has been found as the dominant phytochemical in the essential oil of aromatic herb species in the family Lamiaceae such as oregano and thyme. This compound has been reported to display protective effects in chemically induced breast cancer models in vivo. O. Herrera-Calderon et al. proposed and evaluated the possible mechanism of action of carvacrol by using an in silico study on selected receptors involved in breast cancer progression by docking analysis, molecular dynamics, and drug-likeness studies. Their findings suggest that mTOR signaling pathway could be a possible mechanism of action for its preventive properties on the breast cancer model.

Cynanchum paniculatum (Bge.) Kitag (CP) (also known as dog strangling vine, radix cynanchi paniculata, Shu Changching in Chinese) has been used in conjunction with other medicinal plants to treat cancer in TCM. S.-Y. Yang et al. investigated the anticancer potential of this plant extract against selected breast cancer cell lines with different mutation types (i.e., MDA-MB-231, MCF-7, and ZR-75-1 and SK-Br-3). They found out that negative estrogen receptor and progesterone receptor cells are more sensitive to $\mathrm{CP}$ treatment in terms of direct cytotoxicity, which is not regulated by caspase-3, but highly correlated to MMP-2 regulation. They conclude that $\mathrm{CP}$ displayed effective anticancer potential against selected breast cancer cells through diverse mechanisms of action specifically targeting the inhibition of proliferation of triple negative MDA-MB-231.

The systematic review and meta-analysis of the use of polyherbal traditional Chinese medicine using existing clinical data is also presented in three articles of the special issue.

Cinobufacin is a patent-protected traditional Chinese medicine that has been widely used for the treatment and prevention of breast cancer in China. The medicine is obtained from the skin of toad, Bufo gargarizans that contains diverse components such as toadoxin, dehydroxytoluotoxin, serotonin, and arginine complex. J. Xu et al. reviewed the efficacy and safety of cinobufacin combined with chemotherapy for advanced breast cancer treatment. They found out that the ORR (overall response rate), CBR (clinical benefit rate), and pain relief rate of cinobufacin combined with the chemotherapy group were statistically better than in the chemotherapy group. Cinobufacin combined with the chemotherapy group can also reduce the tumour markers in cancer patients with low negative side effects.

Aidi injection (ADI) is a mixture of selected traditional Chinese herb injections that is composed of the extracts from Panax ginseng C. A. Mey, Astragalus propinquus Schischkin, Acanthopanax senticosus (Rupr. Maxim.) Harms, and Mylabris phalerata Pallas and has been used widely to treat breast cancer in China. Y. Chai et al. conducted the systematic review and meta-analysis of ADI to treat advanced breast cancer. They believe that, based on the few available clinical trials, therapy with ADI in advanced breast cancer patients dramatically alters the overall reaction rate and disease control rate, as well as improves quality of life with few side effects. More randomised control experiments with greater sample sizes, though, should be carried out, as should elucidation of the detailed mechanisms of action (biochemical and molecular level).

Meanwhile, C. Wu et al. investigated and compared the efficacy and safety of a combination of ADI and chemotherapy versus chemotherapy alone in the treatment of 
breast cancer in a systematic review of clinical evidence. From the 20 studies available, they found out that the response rate (RR) and performance status (KPS) in the ADI + chemotherapy group were significantly higher than those of the chemotherapy alone group. They conclude that ADI could act synergistically to enhance the efficacy of chemotherapy drugs with reduced/no additional adverse side effects. They suggest the promotion of ADI as a potent adjunct anticancer drug especially in breast cancer treatment.

In this special issue, 4 bioinformatics research papers were published, particularly by using network pharmacology accompanied with molecular docking application. 1 paper further confirmed the bioinformatics results using a preclinical (in vivo) approach.

In China, compound Kushen injection (CKI) (made from Sophora flavescens and Smilax glabra) has been widely used to treat breast cancer. Its molecular mechanism, however, is unknown. As a result, S. Liu et al. used network pharmacology and molecular docking verification to explore alternative mechanisms of action. The findings revealed that 16 active CKI compounds were recognised, corresponding to 285 putative targets. They discovered that CKI is involved in numerous and complex mechanisms of action in the treatment of breast cancer, including several common cancer pathways, chemical carcinogenesis, oestrogen signalling pathway, TNF signalling pathway, and leukocyte transendothelial migration. Given that this study is primarily focused on data processing, additional biological studies are needed to validate the findings.

FDY003 is an herbal formulation that comprises three herbal preparations, namely, Lonicera japonica Thunberg, Artemisia capillaris Thunberg, and Cordyceps militaris, that have been shown to display potent antitumour effects in different types of cancer cells. As many herbal medicine preparations, the possible mechanism of action is still lacking. Using network pharmacology approaches, H.-S. Lee et al. investigated the mechanisms of FDY003 against breast cancer in the systemic level. They discovered that the herbal mixture modulated cellular processes such as cell proliferation, cell cycle mechanisms, and cell apoptosis, as well as many oncogenic pathways that play important roles in breast cancer pathology.

$\mathrm{K}$. Xiao et al. reported the anticancer potential of Chaihu-Shugan-San, a traditional Chinese medicine that was formulated from seven different kinds of herbal preparations. According to the principles of traditional Chinese medicine, the clinical syndrome of breast cancer refers to the "Liver-Qi" stagnation and Chaihu-Shugan-San is one of the popular treatments for "Liver-Qi" stagnation. In this study, they investigated the possible pathway of Chaihu-ShuganSan in the treatment of breast cancer by network pharmacology. Preliminary results showed that 157 bioactive compounds and 8074 potential drug targets were obtained. Network pharmacology analysis showed that the pathway responsible for the potent anticancer agent includes (but not limited to) mRNA and RNA catabolic processes, telomere organization, apoptosis, cell cycle progression, transcriptional dysregulation, endocrine resistance, and viral infection. They conclude that the treatment of ChaihuShugan-San on breast cancer involves multicomponent, multitarget, and multipathway interactions.

Shuganhuazheng formula (SGHZF) is an anticancer formulation that has been used widely in selected TCM and conventional hospitals in China for many years, especially to treat triple-negative breast cancer (TNBC). B. Wang et al. examined the medicinal effect and mechanism of SGHZF against TNBC using network pharmacology and further verified the efficacy in animal models. They found out that SGHZF has been shown to inhibit the proliferation of breast cancer growth in experimental animal models and the possible mechanism of action might involve the inhibition of Akt and HIF- $1 \alpha$ expression.

One randomised clinical trial was conducted using moxibustion, a type of TCM that involves the burning of moxa, a cone or stick made of ground mugwort leaves, on particular points on the body with the intention to protect health and prevent disease. Previous studies showed that moxibustion may reduce the incidence of cancer and the side effect of chemotherapy. As a result, Y. Ji et al. explored the therapeutic effectiveness of moxibustion for breast cancer patients undergoing adjuvant chemotherapy who have chemotherapy-induced myelosuppression (CIM). They conclude that moxibustion is useful for treating CIM in breast cancer patients undergoing adjuvant chemotherapy, especially in patients undergoing high-dose, long-term, and mixed chemotherapy regimens. Furthermore, moxibustion can reduce the occurrence of SAE (in myelosuppression) and $\mathrm{AE}$ (such as nausea, vertigo, bone, joint, and muscle pain, and incision pain) and improve the compliance and safety of chemotherapy.

A wide range of contributions from a diverse research scope that includes isolation and semipurification of bioactive compounds, preclinical (in vitro and in vivo) studies, possible mechanisms of action using molecular and bioinformatics (network pharmacology and molecular docking) approaches, systematic reviews and meta-analysis of published clinical data, and clinical trials of herbal medicine showed the promising potential of herbal medicine as prevention and therapy in breast cancer.

\section{Data Availability}

All data were obtained from the published studies.

\section{Conflicts of Interest}

The Guest Editors declare that they have no conflicts of interest regarding the publication of the special issue.

\section{Acknowledgments}

The Guest Editors of this special issue acknowledge all contributors to the success of this fantastic publication concerning the use of herbal products as prevention and treatment in breast cancer. In addition to that, the Guest Editors would like to thank all authors of the published articles of this special issue for their respected scientific 
works, reviewers for their comments and suggestions and spending their valuable time to review the articles that made this special issue possible, and the editorial board and the person in charge of this journal for inviting them to host this special issue. The Lead Guest Editors acknowledge the support provided by the Ministry of Higher Education of Malaysia (MOHE) under Fundamental Research Grant Scheme (FRGS), Vot No. 1560 (FRGS/1/2015/WAB01/ $\mathrm{UTHM} / 02 / 1$ ) that encourage scientific research related to complementary and alternative medicine in Malaysia, especially focusing on the use of natural products as prevention and treatment of cancer (breast cancer in this case).

Mohd Fadzelly Abu Bakar Azis Saifudin

Peng Cao

Norhaizan Mohd Esa 